\title{
Study on Mobile Learning Platform based on Wireless communication
}

\author{
Huang Xianying \\ School of computer science and technology \\ Chongqing University of Technology \\ Chongqing, China \\ hxy463@163.com
}

\author{
Cao Qing \\ School of computer science and technology \\ Chongqing University of Technology, \\ Chongqing, China \\ jing5589@163.com
}

\begin{abstract}
The rapid development of mobile technology has brought the change of learning. Mobile learning refers to a new kind of learning for the use of wireless communication technologies and mobile equipments to obtain the educational information, resources, and services. The framework of mobile learning system using wireless communication technology is proposed, a mobile learning support system with multiple wireless terminals is implemented which customs communication protocol to communicate with each other and uses multi-threading technology. The server push learning content to the terminal, learners receive information or to send information to the server through wireless terminal, to feedback information and interaction, and server processes terminal information, data analysis, and then sent to the learner. The system can be applied to a variety of mobile learning occasions, the application shows that the effect of the system effectively reduce the learning environment, and to increase the participation and interest of the learner, improve learning efficiency and learning outcomes.
\end{abstract}

Keywords- Mobile learning, wireless communication, Content push, multi-threaded, communication protocol

\section{INTRODUCTION}

The rapid development of mobile communication technology brought the great transformation of the socioeconomic and the way of people's daily lives. The application of mobile technology in the field of education gave birth to a new form of learning _ mobile learning, making learning everywhere.

Mobile learning is a learning process at any time, any place with the help of computing device, mobile devices using in mobile learning must effectively present learning content and provide the two-way communication between teachers and learners. Ii should have the following characteristics: (1) portability, it has small size, light weight and easy to carry; (2) wireless, it can use without connection; (3) mobility, it can use well in moving. Therefore, the study mobile learning support system based on the wireless handheld device has a very important significance.

\section{The STRUCTURE OF MOBILE LEARNING MODEL}

Mobile learning is based on wireless communication technology. the mobile terminal can access resources on the network, and has brought great convenience to the wireless communication users, so learning to transfer from the computer to the mobile phones and other terminals. However, due to the communication protocol varies between mobile devices and mobile communication networks, the complexity of the hardware, and the diversity of the learning content, mobile learning equipments shows a diverse and complex characteristic, so design of mobile learning devices is very complex.

We first present the overall framework of mobile learning model shown in Figure 1. This model includes one server and multiple clients. Database and mobile learning applications runs on the server, functionality of client is simple, the software in client is installed according to the customer learning needs, the client responses interacts with the service-side application, feedback and interact with the server. Based on this general model, you can develop more and more mobile learning applications without change the overall structure of the learning platform.

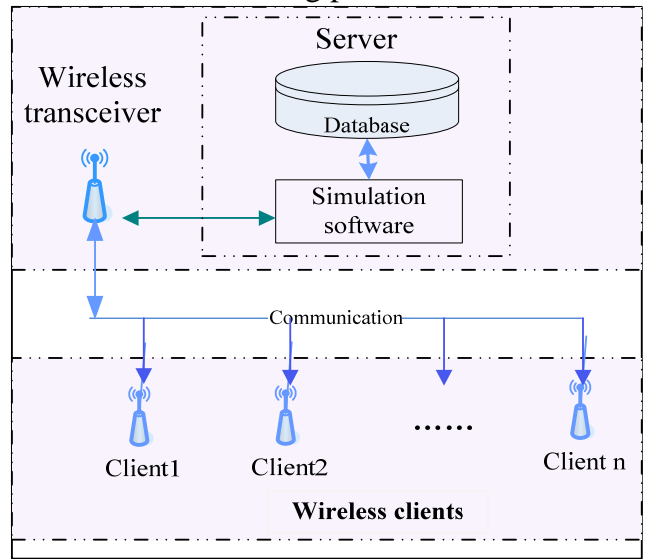

Figure 1 the overall structure of portable learning model

The server is mainly used to process data, control the learning process and handle the experimental results. Controlling the learning process mainly refers to control data exchange between server and client. During the learning, server is responsible for sending learning contents and controlling commands to the client, and collecting results and statistics information from clients.

The client is used by the learner, communications between client and server can use a variety of network communication technology, and client can also be a variety of terminal equipment. The functionality of the client is different from the mobile learning applications. The main module of the learning application runs in server, the client is only responsible for interacting with the server, answering 
relevant information in order to accomplish the learning task together.

\section{DESIGN FOR MOBILE LEARNING PlATFORM}

Following is the design of the software, handheld terminal and communication of the mobile learning system.

\section{A. Design for Mobile Software}

\section{A.1 Modules of the Software}

The platform can run on a number of different applications, so we must consider the independence and cohesion of the module in designing software. The mobile platform includes the following modules: wireless communication module, authentication module, mobile learning application module and results processing module.

The wireless communication module completes the data communication between the handheld terminal and the server. The authentication module complete the

authentication of the students, so that the teacher statistics and analyses the student learning.

The mobile learning application modules are customized according to different learning contents, including parameter setting module and learning controlling module. Parameter setting module is mainly used to set the various environment variables, the learning controlling parameters are different from each other, it is the most important part of the software, It controls the main learning procedure to ensure learning and experimenting orderly until the completion of the learning task, and gets some hardware data and related learning recording data from the learning process.

The results module is mainly used for recording learning results, drawing the diagram of the results, comparing with the history of the learner, and analyzing experiment statistical data of all the students. It plays an important role in assessment and improvement of the learning content and completion.

\section{A.2 Multi-threading mechanism in software}

In the overall structure of the mobile learning platform, server runs the learning applications, it also need communicate to more than one terminal, and this need control strictly each terminal, handle the responded data between terminal and server. These strict controls rely on windows multi-threading technology. The platform software of mobile learning uses multi-threaded event handling mechanism. The software consists of the main thread, message-receiving thread, message-sending thread, messagedispatching thread, message-processing thread, and resultprocessing thread. The main thread is used to coordinating with the threads; message-receiving and message-sending thread is used to receive and send data which include the learning instructions sent from the server, the learning content and the information received from the terminal; message-dispatching thread is used for the received data classification; message-processing thread is the most important thread, it focuses on the data processing, and sends data to message-sending thread. The application runs orderly and controlled effectively by these threads. Multi-threaded event handling mechanism in this system is shown in Figure 5.

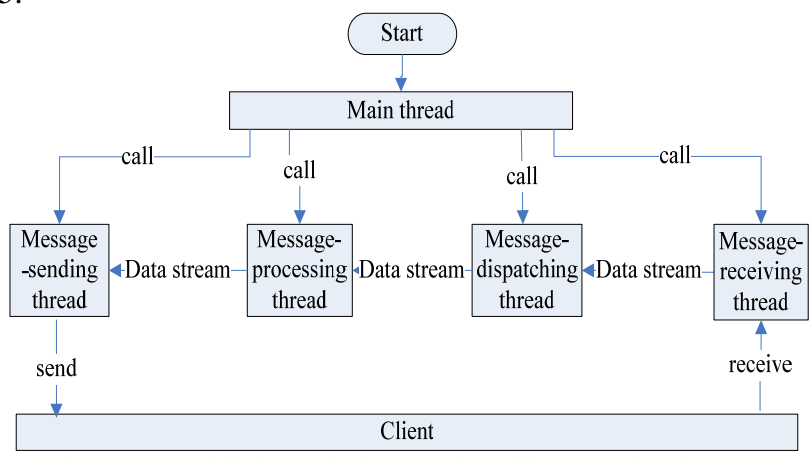

Figure 5 multi-threaded event handling mechanism

After the learning, the result-processing thread is started by the main thread to record the learning results to the database, to draw the results figures so as for the learner and manager to analyze and assess, all information in this learning can output to a word document or printer.

\section{B. Design for Communication}

\section{B.1 Design for Communication Process}

The mobile terminal is passive, the terminal responses appropriately under the protocol only it gets an instruction from the server. Only registered terminal can communicates successfully with the server normally, and completes followup operations. Communication between the terminal and the server must follow the following rules:

(1) Each terminal has a unique ID, the terminal log in when it receives the radio package from the server, and gives its own ID to the server in the same time;

(2) Server can selectively send information to any terminal independently, the other terminal does not recognize this information;

(3) Client can input information only when it receives the input instruction;

(4) Only one learning process is completed, another can be started. That is, when the terminal receives an end instruction of one learning process, it can start another one;

(5) If the terminal does not response after a given amount of time, the server will consider this terminal give up, it will exit the learning process, the server is no longer receive data from this terminal.

\section{B.2 Design for mobile platform communication}

Communication between the server and client uses wireless communication technology, so a wireless transceiver is configured on the server, which is inserted into the host USB port when used. It is responsible for sending the learning content to wireless terminal through broadcasting and receiving the information from terminals, its working principle is shown in Figure 3.

Communication between the server and client needs protocols, which refers to communication rules enabling mutual understanding by communicating parties. Error may be emerging while data transmitting and receiving by the outside interference because of the characteristics of the wireless transceiver module. So it is needed to make protocol to ensure that the receiver can correctly receive the data from 
the transmitter, and to determine whether the received data is correct. The protocol is divided into three layers which is physical layer, data link layer and application layer.

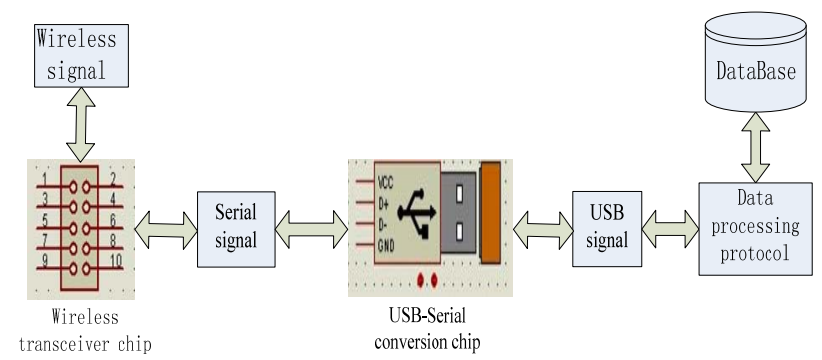

Figure 3 Communication mode of the platform

Physical layer uses a dedicated radio frequency chip to complete the data transmission, distribution and data validation, which is the XD-RF4-13-S chip in the system.

Data link layer provides reliable wireless data transferring, it defines the data frame format and the meaning of each byte. Data frame includes seven fields, a data field and six flag field occupied 8 bytes, as shown in Table 1, including: 0xFF;

(1) The first byte of the frame is starting tag, fixed to

(2) Bytes 2, 3 and 4, 5 are the receiver ID and sender ID, which are two bytes respectively, the server is fixed at $0 \times 0000$, ID number of each terminal is in range of 0x0001 0xfde8 (65000-252);

(3) Byte 6 is the number of selected learning applications;

(4) Byte 7 and 8 are respectively the data length of valid data and descriptive data;

(5) The actual data is after the 8 bytes. Data is divided into non-broadcast data and broadcast data. When the receiver ID is OXFFFF in the frame, the data is a broadcast packet, the other value of the receiver ID indicates that the data is valid for the specific terminal. Chinese characters in the data stream are presented with the GB2312 standards codes, letters and numbers are with ASCII codes.

Application layer refers to a variety of practical mobile learning applications. The functions include Initialization, displaying all the current successful handshaking terminal state, and determining which terminal is interacted with server. Server can receive data from each terminal, do the final summary of all learners, and analyze the results, output the graphical results.

\section{Design for mobile terminal hardware}

The terminal need to deal with a lot amount of display information and reads more key values from I/O port. So chip in the terminal requires more than 40 pin so as to expand and control directly, and reduce the circuit complexity and power consumption.

The terminal needs more interactions with the server, it used frequently, so power requires detecting, and has digital/analog conversion module. The terminal must deal with the complex logic judge and show the result, complete the communications process control, data encryption and decryption, data packed and unpacked, the keyboard scan processing during the learning process. Therefore, the chip must have a large program memory space.

In view of the above considerations we chose the microcontroller STC12C5A60S2 with the UART serial communication as terminal control module.

The terminal is composed of wireless transceiver module, keyboard module, power module, display module, downloading module and master controlling modules. Wireless transceiver module is responsible for receiving information from the server, power module is responsible for providing power, keyboard module is responsible for inputting signal to the controlling module, controlling module receives and processes the input information and display in the display module, downloading module is connected with controlling module two-way, the program which needs to be updated in control module is updated by downloading module.

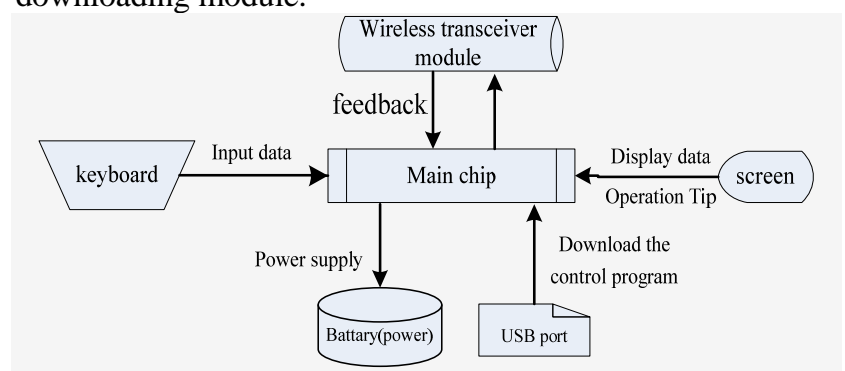

Figure 4 hardware function structure of the platform

\section{APPLICATION OF THE PLATFORM}

Based on this general mobile learning platform, we can design a variety of mobile learning application or mobile experimental simulation system. Following gives the auction learning process in experimental economics and experimental simulation to verify the function of the platform.

The learning process is controlled by the teacher. The screen is divided into the main control window and a realtime information window of learner, the learners can only see the real-time information window; they cannot see the control window. In figure 6, the upper part is the parameters; the left part is the learner's ID, the right part is the real-time learning contents.

The result processing includes drawing a chart, outputs to the word, printouts and other functions, this function is also divided into the teacher's control window and learner's display window, shown in Figure4. 




Figure 3 Screen of one application

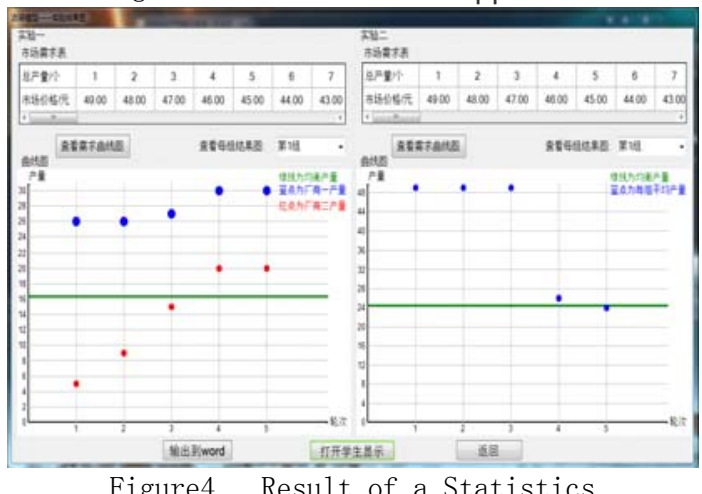

V. SUMMARY

This paper describes the research and design of the mobile learning platform based on wireless communication technology. The client is designed as a hand-held terminal taking into account the data traffic between the client and server in the learning process is not large, and also taking into account the ease of use and cost. The server and terminal communicates with each other using wireless communication technology via custom protocol. The multithreaded programming techniques are used in software design to solve the conflict or multi-client signal and the bottleneck problem of signal processing in the platform.
Based on the platform, you can add different mobile learning application or experimental programs. In order to improve the environmental adaptability and the breadth of applications, we control each terminal strictly using wireless communication technology, and set various parameters of the learning environment and learning process. Finally, we test the mobile learning system through "experimental economics" learning applications, it greatly enhances the efficiency of learning, improve the learning effect, improve the experimental environment, improve the learners' interest, learners have a real experience in learning.

\section{ACKNOWLEDGEMENT}

This research was financially supported by the committee of Chongqing Education (Grant NO. KJ100821)

\section{REFERENCES}

[1] Sun Liping, Electromagnetic shielding design in electronic equipment and applications in testing the composite materials shielding, manufacturing automation, 2011.5

[2] Wang Haibo; Yu Guo, etc. Validation with dynamic thread creation and exit of the multi-threaded programs. Mini-Micro Systems, 2010.8,P33-35

[3] Zhu Lili. Scene perception-based mobile learning application research, modern educational equipment, 2012.15, P83-85

[4] Ren Yan. Smart phone-based mobile learning system design, Gansu Science and Technology, 2012.18, P17-18

[5] Wang Qiang, Zhen shiyu, Song Zhiming. The Design and Implementation of Interactive Micro Reader Based on Android, Modern educational technology, 2012.9, 89-92

[6]Yu Yongsheng. On the current wireless hotspot technology and trends in wireless communications, China water, 2010.10,P54-56

[7]Liu Juanjuan, etc., wireless features' design and implementation in the wireless handset, Computer Engineering, 2008.9, P89-92

[8]Zhu Qi, Li Zhaojun, etc. Study of English teaching supporting system based on handheld devices, Chinese information technology in education, 2009.10, P66-68

[9] Wang Jianxin, Remote monitoring technology development and trends, Foreign Electronic Measurement Technology, 2008.9, P9-12

[10] Fang Haiguang,Mao Ying, Liu Jun. Research of System Support Environment of Mobile Learning. Modern educational technology, 2011.21, P15-20 